\title{
Non-echo-planar diffusion-weighted MRI in cholesteatoma: One typical case, one atypical case and one rare false positive finding
}

\author{
Martin W. Huellner ${ }^{1^{*}}$, Eva Novoa ${ }^{2}$, Manfred Kessler ${ }^{1}$, Thomas C. Treumann ${ }^{1}$, \\ Thomas E. Linder ${ }^{2}$ \\ ${ }^{1}$ Department of Radiology and Nuclear Medicine, Lucerne Cantonal Hospital, Lucerne, Switzerland; \\ *Corresponding Author: martin.huellner@usz.ch \\ ${ }^{2}$ Department of Otorhinolaryngology, Head and Neck Surgery, Lucerne Cantonal Hospital, Lucerne, Switzerland
}

Received 20 September 2013; revised 20 October 2013; accepted 3 November 2013

Copyright (C) 2013 Martin W. Huellner et al. This is an open access article distributed under the Creative Commons Attribution License, which permits unrestricted use, distribution, and reproduction in any medium, provided the original work is properly cited.

\begin{abstract}
We present two cases of cholesteatoma and one false positive finding in non-EP DW MRI in order to highlight the differential diagnosis in imaging and emphasize the need to discuss the findings with the otologic surgeon. The first case demonstrates different MRI signal patterns encountered in a patient with cholesteatoma. The second report is a rare case of supralabyrinthine cholesteatoma with atypical clinical presentation. The third case presents a rare but important false positive finding in non-EP DW MRI. Clinical and imaging findings are discussed taking into account the current literature.
\end{abstract}

Keywords: Cholesteatoma; Non-EP DW MRI; Diffusion-Weighted Imaging

\section{INTRODUCTION}

A simple definition of cholesteatoma is "wrong skin at the wrong place with retention of keratin". The expansive characteristics of the keratinizing squamous epithelium lead to sharp bony erosions that are easily appreciated on computed tomography (CT) scans. However, CT cannot confirm the true presence of a cholesteatoma mass since it differentiates poorly between soft tissue lesions. Recently, non-echo planar diffusion-weighted MR imaging (non-EP DW MRI) has gained much attention due to its short acquisition time and high sensitivity and specificity for detecting even small cholesteatomas of $2-3 \mathrm{~mm}$ in size [1]. Whereas this fast sequence may be sufficient to screen for residual cholesteatoma in canal wall-up procedures, we encourage using additional T2- and T1-weighted sequences in cases of primary diagnosis of a soft tissue lesion within the temporal bone. We present three cases to highlight the differential diagnosis in imaging and emphasize the need to discuss the findings with the otologic surgeon.

\section{CASE REPORTS}

\subsection{Case 1}

A 32-year-old male patient presented with a history of ear pain and recurrent hearing loss on the left side for 2 months. Ten years ago, a pathological otoscopy was reported by a general physician, but no further follow-up was recommended. At present, a polyp and a grey mass behind an epitympanic retraction pocket were observed. Audiometry revealed an air-bone gap of $10-25 \mathrm{~dB}$. A primary acquired epitympanic cholesteatoma was suspected and its extent had to be assessed.

CT scans of the temporal bone revealed subtotal obliteration of the left middle ear and partial obliteration of the antral mastoidal cells (Figure 1(a)). The ossicle chain was embedded into the opacity. Bony erosion was suspected. MRI work-up provided further differentiation of the soft tissue mass in the middle ear. Within the mass, a bright signal spot on non-EP DWI (Figure 1(b)) was interpreted as cholesteatoma. The non-EP DWI sequence was correlated with a heavily T2-weighted transverse CISS (constructive interference in steady state) sequence. The cholesteatoma proved to be the tissue lateral to the ossicles and in the attic and did not enhance contrast medium on a T1-weighted delayed post-gadolinium sequence (Figure 1(c)). In contrast, the tissue medial to the ossicles and lateral to the promontory showed distinct contrast enhancement in the absence of diffusion restriction, strongly suggestive of inflammatory tissue. The 


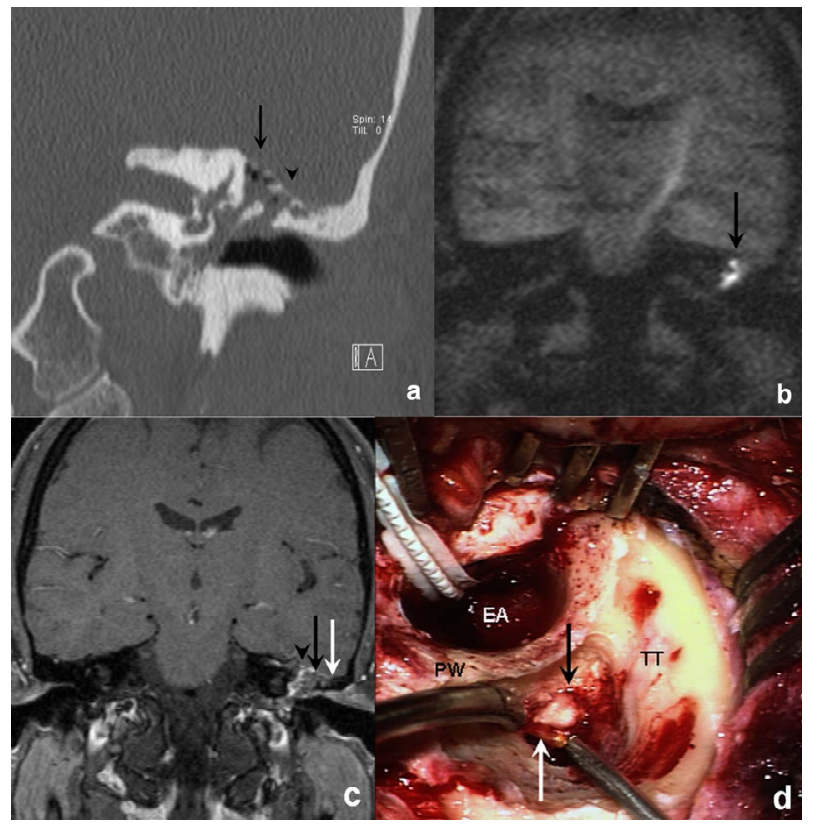

Figure 1. (a) NECT (coronal reformat, W/C 3500/600, 250 $\mathrm{mAs}$, voxel size $0.6 \times 1 \times 1 \mathrm{~mm}$ ): Ossicle chain embedded in soft tissue (arrow) and partial obliteration of the antral mastoidal cells (arrow head); (b) Non-EP DWI (coronal, b-value: 1000s/mm², voxel size $1.5 \times 1.1 \times 3 \mathrm{~mm}$, TR $2000 \mathrm{~ms}$, TE 107 $\mathrm{ms}$ ): Bright signal spot in the left middle ear suggestive for cholesteatoma (arrow); (c) Contrast-enhanced T1w with fat saturation (coronal, voxel size $1.0 \times 0.8 \times 2.0 \mathrm{~mm}$, TR 533ms, TE $13 \mathrm{~ms}$, gadolinium-DTPA): Non-enhancing cholesteatoma lateral to the ossicles (arrow). Enhancing inflammatory tissue medial to the ossicles (arrow head). Fluid retention in mastoid cells (white arrow); (d) Intraoperative view of the cholesteatoma (black arrow) obliterating the mastoidal antrum. A cholesterol granuloma (white arrow) is identified just below. EA: external auditory canal, PW: posterior wall (black arrow head), TT: tegmen tympani.

mastoidal cells were obliterated and showed bright signal on $\mathrm{T} 2 \mathrm{w}$ without contrast enhancement, corresponding to fluid retention. The findings were confirmed surgically. A closed mastoido-epitympanectomy (MET) with canal-, myringo- and ossiculoplasty was performed and the cholesteatoma was completely removed (Figure 1(d)).

\subsection{Case 2}

A male patient with primary acquired cholesteatoma underwent open MET at the age of 7. A second look procedure with ossiculoplasty using a titanium-incus interposition was performed one year later. Regular follow-up examinations revealed a dry open cavity without clinical suspicion of recurrent cholesteatoma. At the age of 14, he presented with an acute facial paresis on the left side, rapidly progressing to an almost complete paralysis (Fisch score 15, House-Brackmann score V). Suspecting acute Bell's palsy, a seven days trial of $100 \mathrm{mg}$ prednisolone per day and $1 \mathrm{~g}$ valacyclovir tid were given, with minimal improvement in facial function.

CT scans of the temporal bone showed soft tissue densities within an osteolysis in the apex of the left temporal bone, as well as in the widened knee of the facial canal (Figure 2(a)). Corresponding non-EP DWI and T2weighted sequences showed a bright spot in the apex of the temporal bone, and a smaller, less bright spot in the facial knee (Figure 2(b)), suspicious of a supralabyrinthine cholesteatoma with portions in the petrosal apex and in the facial knee. Thereby, the initial diagnosis of Bell's palsy was revised. A combined transtemporaltransmastoidal approach with revision of the open MET and complete removal of the cholesteatoma, which was located medial to the geniculate ganglion and compressed the labyrinthine segment of the facial nerve, was performed 16 days after the onset of the palsy (Figures 2(c) and (d)). The less bright spot in the petrosal apex turned out to be scar tissue with polypoid swelling. Upon discharge six days later, facial nerve function had recovered to a House-Brackmann score II and recovered completely one month later.

\subsection{Case 3}

A 41 year old male with a history of three previous ear

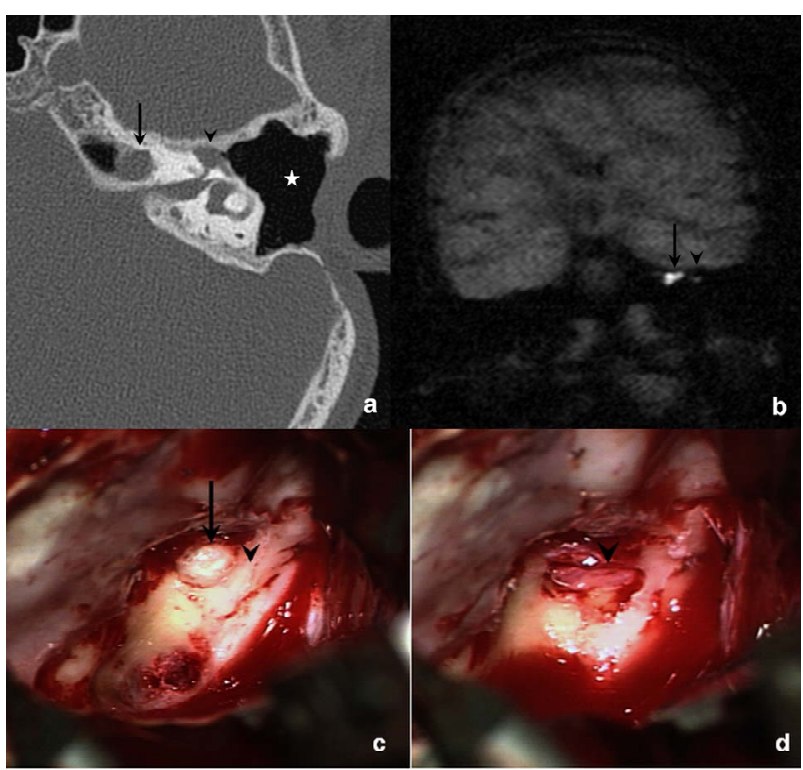

Figure 2. (a) CT (axial, W/C 3500/600, $250 \mathrm{mAs}$, voxel size $0.6 \times 1 \times 1 \mathrm{~mm}$ ): Osteolysis in the petrous apex (arrow) and widening of the facial canal (arrowhead). Open cavity after MET procedure (white asterisk); (b) Non-EP DWI (coronal, b-value $1000 \mathrm{~s} / \mathrm{mm}^{2}$, voxel size $1.5 \times 1.1 \times 3 \mathrm{~mm}$, TR $2000 \mathrm{~ms}$, TE $107 \mathrm{~ms}$ ): Bright signal spot in the petrous apex (arrow) and smaller, less bright spot in the facial knee (arrow head); (c) Intraoperative view before removal of the cholesteatoma: the labyrinthine segment of the facial nerve (arrow head) is compressed by the cholesteatoma (arrow); (d) Intraoperative view after removal of the cholesteatoma: the labyrinthine segment of the facial nerve (arrow head). 
surgeries within the last ten years for recurrent cho lesteatoma, complained of recent pressure on the right ear. On inspection, a flat white mass was seen covering the area of the lateral semicircular canal and stapedial footplate. A residual cholesteatoma within an open cavity was suspected, and the patient was referred for non-EP DW MRI. On T2-weighted images and on non-EP DW images, the mass had a fairly bright signal, suggestive of recurrent cholesteatoma (Figures 3(c) and (d)). After MRI, the cavity was extensively cleaned by the otologic surgeon, and the white incrustation could be removed from the surface of the lateral semicircular canal and the

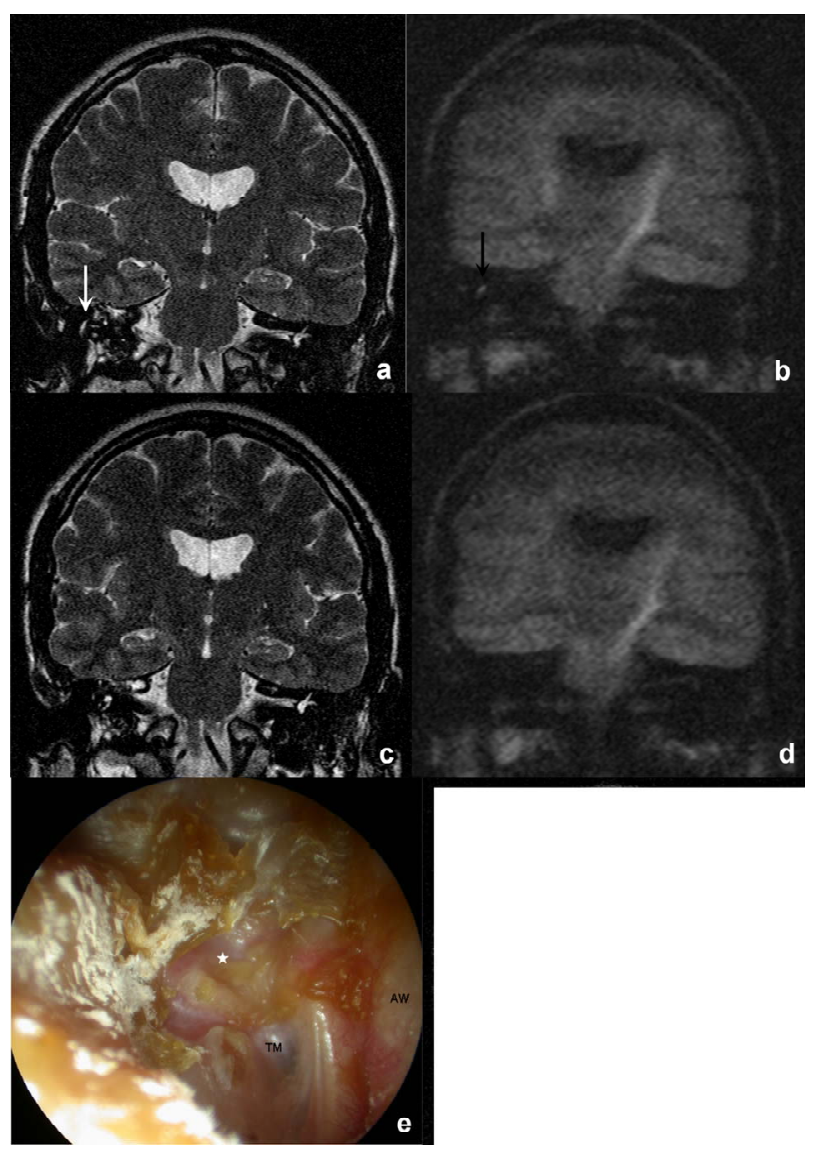

Figure 3. (a) $\mathrm{T} 2 \mathrm{w}$ (coronal, voxel size $2 \times 0.7 \times 0.4 \mathrm{~mm}$, TR $4930 \mathrm{~ms}$, TE $124 \mathrm{~ms}$ ): Bright signal lesion at the medial wall of the left middle ear (white arrow); (b) Non-EP DWI (coronal, b-value: $1000 \mathrm{~s} / \mathrm{mm}^{2}$, voxel size $1.5 \times 1.1 \times 3 \mathrm{~mm}$, TR $2000 \mathrm{~ms}$, TE $107 \mathrm{~ms}$ ): Bright to intermediate signal lesion at the medial wall of the left middle ear (arrow); (c) T2w (coronal, voxel size $2 \times 0.7 \times 0.4 \mathrm{~mm}$, TR $4930 \mathrm{~ms}$, TE $124 \mathrm{~ms}$ ): Repeat MRI after removal of the middle ear incrustation: the signal at the middle ear wall has vanished; (d) Non-EP DWI (coronal, b-value: 1000 $\mathrm{s} / \mathrm{mm}^{2}$, voxel size $1.5 \times 1.1 \times 3 \mathrm{~mm}$, TR $2000 \mathrm{~ms}$, TE $107 \mathrm{~ms}$ ): Repeat MRI after removal of the middle ear incrustation: the signal at the middle ear wall has vanished; (e) View on inspection a few months after the MRI: Right open mastoid cavity containing debris and layers of keratin close to the lateral semicircular canal and promontory (asterisk). AW: anterior wall of the external auditory canal, TM: neotympanon. promontory, lateral to the atelectatic but intact tympanic membrane. It resembled layers of a keratinous substance mixed with cerumen. In order to prove that there was no residual “cholesteatoma”, the MRI was repeated, and, as was expected, the suspicious signal was gone (Figures 3(c) and (d)). No surgery was performed. On follow-up clinical investigation one month later, reformation of cerumen could be seen (Figure 3(e)).

\section{DISCUSSION}

Cholesteatomas are either primary or secondary acquired chronic inflammatory lesions of the middle ear and mastoid with ingrowth and retention of keratin, or may present as congenital lesions behind an intact tympanic membrane. The destructively growing and expansive keratinizing squamous epithelium requires complete surgical removal. The pathogenesis of cholesteatoma as well as the incidence and reasons for residual and recurrent lesions are still subject of research and debates [2].

While CT continues to be the best imaging modality for preoperative planning $[3,4]$, differentiation of cholesteatoma from fluid or inflammatory tissue is impossible by tissue density on CT alone. If a mass is found in the typical location or if bone destruction is present, a cholesteatoma can be assumed.

MR imaging is superior to CT because of its possibilities to further characterize tissue. For the detection of cholesteatoma, diffusion weighted imaging (DWI) has proved to be of great value. In DWI, the intensity of the magnetic resonance signal depends on the stochastic Brownian molecular motion, i.e. on the self-diffusion capability of the excited spins [5]. Water molecules in cholesteatomas are restricted in their diffusion capability and contribute to high signal in the images. The standard technique of DWI is echo-planar (EP), commonly used for the early detection of cerebral infarction. In the temporal bone however, the value of EP DWI is limited because of susceptibility artifacts. Here, the appropriate technique is non-EP DW imaging which has a slightly longer acquisition time but much less susceptibility artifacts and higher signal for cholesteatoma. Non-EP DWI has a high sensitivity and specificity for cholesteatoma [5-7]. Occasionally, small keratin pearls of less than 2 - 5 $\mathrm{mm}$ may cause false-negative results $[3,8,9]$. False-positive results in DWI have been reported for susceptibility artifacts, scar tissue, bone powder, abscess, and chordoma $[3,8,10]$. To our best knowledge, false-positive results by keratinous material in non-EP DWI have not been reported yet.

We showed a valuable example of a false-positive case in a postoperative patient. In case 3 we assumed that keratin-like material of a certain chemical constitution may mimic cholesteatoma by generating a bright signal in non-EP DWI. To avoid misinterpretation on MRI, we 
suggest inspecting and cleaning the ear on a short-term basis before referring the patient to the MRI examination, especially during follow-up after cholesteatoma surgery and in patients known to produce retention of dry ceruminal wax in their ear canal. A possible group of patients at risk of such a false-positive finding are those with insufficient or narrow meatoplasties, as the complete radical cavity can only be overseen poorly, and cleaning of debris remains a problem. The number and frequency of second look operations after canal wall-up procedures has markedly declined after the introduction of non-EP DW MRI which may be performed $1-1 \frac{1}{2}$ years after initial surgery to exclude residual cholesteatoma behind a reconstructed intact tympanic membrane.

Since DWI images have a low spatial resolution almost without anatomic landmarks in the temporal bone, a high-resolution T2-weighted sequence is required to correlate DWI findings with the patient's anatomy. A delayed post-gadolinium T1-weighted sequence is not needed for the detection of cholesteatoma as it does not yield significant increase in sensitivity or specificity if obtained in addition to a non-EP DWI sequence [11]. One cannot rely on diffusion signal alone, as false-positives may occur in certain conditions. In case of a negative non-EP DWI sequence, an axial T1-weighted sequence with and without gadolinium should be obtained additionally to rule out other pathologies like inflammation or tumor. The prevalence of both cholesteatoma and inflammation is seen quite frequently since the cholesteatoma may block the antrum and lead to sterile inflammatory changes in the mastoid mucosa, or the cholesteatoma sac may be infected and the miscellaneous bacterial flora induces an inflammatory response in the middle ear.

\section{CONCLUSION}

The primary diagnosis of cholesteatoma is based on the typical otoscopic findings. A high level of suspicion is required for diagnosis in post-operative patients with an intact and reconstructed tympanic membrane and canal wall, in patients with a supra- or infralabyrinthine extension of the cholesteatoma, being not accessible by simple otoscopy, and in previously operated ears with a narrowed canal entrance, complicating the inspection of the cavity. Radiological imaging helps to confirm the diagnosis, provides a differential diagnosis and facilitates surgical planning. Non-EP diffusion MRI can be used to identify and localize cholesteatoma with high sensitivity and specificity [5,7]. False-negative results can be obtained in foci smaller than 2 - $5 \mathrm{~mm}[1,9,10]$. False-positive results may be caused by scar tissue $[3,8]$, bone powder [10] and keratinous material embedded in the debris of the radical cavity. The imaging gold standard remains non-EP DWI [1,6,9,12]. Echo-planar DWI should be abandoned. For anatomic correlation, a high-resolution T2-weighted image is required. If the non-EP DWI signal does not suggest cholesteatoma, T1-weighted nonenhanced and gadolinium-enhanced sequences should be added to differentiate inflammatory tissue or tumor from fluid.

\section{REFERENCES}

[1] De Foer, B., Vercruysse, J.P., Bernaerts, A., Deckers, F., Pouillon, M., Somers, T., et al. (2008) Detection of postoperative residual cholesteatoma with non-echo-planar diffusion-weighted magnetic resonance imaging. Otology \& Neurotology, 29, 513-517. http://dx.doi.org/10.1097/MAO.0b013e31816c7c3b

[2] Louw, L. (2010) Acquired cholesteatoma pathogenesis: Stepwise explanations. The Journal of Laryngology \& Otology, 124, 587-593. http://dx.doi.org/10.1017/S0022215109992763

[3] Jeunen, G., Desloovere, C., Hermans, R. and Vandecaveye, V. (2008) The value of magnetic resonance imaging in the diagnosis of residual or recurrent acquired cholesteatoma after canal wall-up tympanoplasty. Otology \& Neurotology, 29, 16-18.

http://dx.doi.org/10.1097/MAO.0b013e31815dbae8

[4] Mafee, M.F. (1993) MRI and CT in the evaluation of acquired and congenital cholesteatomas of the temporal bone. Journal of Otolaryngology, 22, 239-248.

[5] Dietrich, O., Biffar, A., Baur-Melnyk, A. and Reiser, M.F. (2010) Technical aspects of MR diffusion imaging of the body. European Journal of Radiology, 76, 314-322. http://dx.doi.org/10.1016/j.ejrad.2010.02.018

[6] De Foer, B., Vercruysse, J.P., Pilet, B., Michiels, J., Vertriest, R., Pouillon, M., et al. (2006) Single-shot, turbo spin-echo, diffusion-weighted imaging versus spin-echoplanar, diffusion-weighted imaging in the detection of acquired middle ear cholesteatoma. American Journal of Neuroradiology, 27, 1480-1482.

[7] Patel, M.R., Klufas, R.A., Alberico, R.A. and Edelman, R.R. (1997) Half-fourier acquisition single-shot turbo spinecho (HASTE) MR: comparison with fast spin-echo MR in diseases of the brain. American Journal of Neuroradiology, 18, 1635-1640.

[8] Aikele, P., Kittner, T., Offergeld, C., Kaftan, H., Huttenbrink, K.B. and Laniado, M. (2003) Diffusion-weighted MR imaging of cholesteatoma in pediatric and adult patients who have undergone middle ear surgery. American Journal of Roentgenology, 181, 261-265. http://dx.doi.org/10.2214/ajr.181.1.1810261

[9] Huins, C.T., Singh, A., Lingam, R.K. and Kalan, A. (2010) Detecting cholesteatoma with non-echo planar (HASTE) diffusion-weighted magnetic resonance imaging. Otolaryngology-Head and Neck Surgery, 143, 141-146. http://dx.doi.org/10.1016/j.otohns.2010.02.021

[10] Dubrulle, F., Souillard, R., Chechin, D., Vaneecloo, F.M., Desaulty, A. and Vincent, C. (2006) Diffusion-weighted MR imaging sequence in the detection of postoperative recurrent cholesteatoma. Radiology, 238, 604-610. 
http://dx.doi.org/10.1148/radiol.2381041649

[11] De Foer, B., Vercruysse, J.P., Bernaerts, A., Meersschaert, J., Kenis, C., Pouillon, M., et al. (2010) Middle ear cholesteatoma: Non-echo-planar diffusion-weighted MR imaging versus delayed gadolinium-enhanced T1-weighted MR imaging-value in detection. Radiology, 255, 866-872. http://dx.doi.org/10.1148/radiol.10091140
[12] Schwartz, K.M., Lane, J.I., Bolster Jr., B.D. and Neff, B.A. (2010) The utility of diffusion-weighted imaging for cholesteatoma evaluation. American Journal of Neuroradiology, 32, 430-436.

http://dx.doi.org/10.3174/ajnr.A2129

\section{ABBREVIATIONS AND ACRONYMS}

\section{AW \\ CISS \\ CT \\ DTPA \\ DWI \\ EA \\ MET \\ MR \\ NECT \\ PW \\ $\mathrm{T} 1 \mathrm{w}$ \\ $\mathrm{T} 2 \mathrm{w}$ \\ TE \\ TM \\ TR \\ TT \\ $\mathrm{W} / \mathrm{C}$}

Non-EP DW MRI
Anterior wall

Constructive interference in steady state

Computed tomography

Diethylenetriaminepentaacetate

Diffusion-weighted imaging

External auditory canal

Mastoido-epitympanectomy

Magnetic resonance

Non-enhanced computed tomography

Non-echo planar diffusion-weighted magnetic resonance imaging

Posterior wall

T1-weighted

T2-weighted

Time to echo

Neotympanon

Time to repeat

Tegmen tympani

Width/center 